\title{
TRANSIENT INDUCED GLOBAL RESPONSE SYNCHRONIZATION
}

\author{
WILLIAM SULIS \\ Collective Intelligence Laboratory, McMaster University, Canada.
}

\begin{abstract}
Synchronization has a long history in physics where it refers to the phase locking of identical oscillators. This notion has been applied in biology to such widely varying phenomena as the flashing of fireflies and the binding problem in the brain. The relationship between neural activity and the behaviour of the organism is complex and still poorly understood. There have been attempts to explain this using the notion of synchronization, but the participating neurons are fungible, their activity transient and stochastic, and their dynamics highly variable. In spite of this, the behaviour of the organism may be quite robust. The phenomenon of transient induced global response synchronization (TIGoRS) has been used to explain the emergence of stable responses at the global level in spite of marked variability at the local level. TIGoRS is present when an external stimulus to a complex system causes the system's responses to cluster closely in state space. In some models, a 10\% input sample can result in a concordance of outputs of more than $90 \%$. This occurs even though the underlying system dynamics is time varying and inhomogeneous across the system. Previous work has shown that TIGoRS is a ubiquitous phenomenon among complex systems. The ability of complex systems exhibiting TIGoRS to stably parse environmental transients into salient units to which they stably respond led to the notion of Sulis machines which emergently generate a primitive linguistic structure through their dynamics. This paper reviews the notion of TIGoRS and its expression in several complex systems models including driven cellular automata, cocktail party and dispositional cellular automata.
\end{abstract}

Keywords: cellular automata, cooperation, emergence, synchronization, TIGoRS.

\section{INTRODUCTION}

Conflict and competition are dominant themes in the social and economic sciences. The vast majority of models in economics are predicated on the assumption that the agents involved in economic transactions are all competing for a limited set of resources and capital. Likewise, many models of group behaviour in the social sciences also make a presumption of competition between the group members, particularly when the members are themselves large social groups such as ethnic populations or nations. Nevertheless there is much in the life of individuals that involves at least some degree of cooperation. While conflict may form a prominent aspect of human designs, cooperation abounds in the natural world. Organisms provide quintessential examples of cooperative systems. Symbiosis between organisms provides another set of examples of cooperation. Social insect colonies provide examples of cooperation both within colonies and sometimes with other species [1].

The most extreme form of cooperative behaviour, yet paradoxically the simplest dynamically, can be seen in the synchronization of the activities of different agents engaged in some task. The actions of two men sawing down a tree using a long saw, or driving spikes into a railway tie, exhibit a form of phase locked synchronization. There is little or no freedom in the actions of each member of the dyad. This type of extreme cooperation resembles the traditional concept of synchronization in classical physics where the prototypical example is of two pendulums sitting on the same mantle piece, 
set in motion separately and whose oscillations gradually adjust until they come to move in lock step (possibly with a difference in phase). Synchronization does not always involve external behaviour. Many studies have been carried out looking for synchronization between unconscious physiological processes such as heart rate and galvanic skin responses [2].

Phase locked synchronized behaviour is much easier to study than cooperative behaviour on the whole since the behaviour of the synchronized system is overtly constrained. This is not true of synchronization phenomena in general and certainly not true of cooperative behaviour where the only constraint may rest with the goal of the behaviour and not the specifics of the behaviour itself.

Phase locked synchronization provides too severe a constraint to be useful for understanding cooperative behaviour among humans. A more general form of synchronization may be observed in the action of a symphony orchestra. This is certainly an example of cooperation among a group of individuals. No single member of the orchestra can perform an orchestral work. Only the ensemble has this capability. Performers play different instruments and produce different musical lines, yet for the work to be musically coherent and consistent they must follow the score with near perfect timing in the coordination of their parts. In that sense their activities are synchronized even though they are not identical.

Synchronization has been suggested as one means by which the brain is able to integrate information from different sensory domains into a coherent experience [3,4]. Unfortunately synaptic activity is noisy and unreliable [5] and neurons generate rather noisy and unreliable rate or frequency modulated signals [6,7]. Their responses appear to be meta-stable (context dependent) [8-10].

Stigmergy (the incitement to work by the products of work) in collective intelligence systems provides another example in which there is context-dependent cooperation at the colony level capable of producing complex nest constructions, yet the behaviour of individual members of the systems appears to be random and uncorrelated [11]. Dynamic networks, in which the network describing the interactions among participating agents is in continual flux, provide another challenge to the modelling of cooperative behaviour, requiring concepts such as compatibility and sociability [12].

These examples suggest that in a complex hierarchical system there may be patterned behaviour appearing at one level, generated by the activity of system components at a lower level, in the absence of any fixed relationship between these two levels of activity. The global patterns are emergent from the interactions of the system's agents with their environment and among themselves, and these interactions are spontaneous, contextual, emergent, and not pre-programmed. Although humans tend to program patterned responses into the behaviour of their constructions, this need not be true of naturally occurring computational systems [13].

The search for a form of cooperative behaviour which possessed the characteristics of being spontaneous, contextual and emergent, while at the same time constrained enough to analyze and general enough to be salient for complex systems led to the discovery of transient induced global response synchronization (TIGoRS).

\section{TIGORS}

TIGoRS was discovered while studying the behaviour of a variety of formal complex systems models (tempered neural networks, cellular automata) interacting with a dynamic environment [14-18]. The behaviour of these systems in the presence of external stimulation proved to be quite distinct from that under autonomous conditions. TIGoRS refers to the phenomenon in which a transient stimulus applied to a dynamical system produces a set of responses which cluster more closely in pattern space than would be expected based upon random chance alone. In many models, these responses clustered in spite of variations in the initial states of the system, initial rule configurations, 
final rule configurations, variability in the agents receiving external stimulation, variability in the sampling of the external transient. Nevertheless a stable correspondence appeared between an external transient (stimulus) and the subsequent global behaviour of the system (responses). This correspondence depended upon symmetry and complexity of the input stimulus and the strength of the stimulus measured as the percentage of agents of the system receiving direct stimulation by the external transient.

Most of the research into TIGoRS has been carried out using cellular automata, which are discrete space, discrete time complex systems composed of individual cells capable of being in any of a set of states, each of which is assigned a fixed neighbourhood of cells with which they interact, and where each subsequent state is determined by a function of its current state and the states of all cells within its local neighbourhood. For example, a 2 state, 3 neighbour cellular automata consists of a set of cells arranged around a circular lattice. Each cell can exist in one of 2 states, 0 or 1 . At time step n, the cell $i$ determines its state $a_{i}^{n+1}$ at time step $\mathrm{n}+1$ by entering its state value and those of its adjacent neighbours into a function $f$, hence $a_{i}^{n+1}=f\left(a_{i-1}^{n}, a_{i}^{n}, a_{i+1}^{n}\right)$.

Each neighbourhood is described by a triple of states $(x, y, z)$ where the value of $x, y, z$ may be 0 or 1. We may encode each neighbourhood as a number from 0 to 7 using the formula: $N_{(x, y, z)}=x+2 * y+2^{2} * z$. Each vector $\left(a_{0}, a_{1}, \ldots a_{7}\right)$ corresponds to a rule as follows: if the neighbourhood of a cell $k$ is $(x, y, z)$ having the value $n$, then the next state of $k$ will be the value $a_{n}$. Each rule is given as a number: $a_{0}+2 * a_{1}+2^{2} * a_{2}+\ldots 2^{7} * a_{7}$.

The Hamming distance $d$ (the total number of sites discordant between two patterns) is a common metric used to study cellular automata patterns. The greater the number of differences between two patterns, the greater their Hamming distance. Let the transients induced in a system $\sum$ under a transient stimulus $\eta$, starting from two distinct initial conditions, be denoted $\Psi_{\eta}, \Psi_{\eta}^{\prime}$. TIGoRS was originally defined to be present whenever $d\left(\Psi_{\eta}, \Psi_{\eta}^{\prime}\right)<\frac{1}{2} d\left(\operatorname{rand}\left(\Psi_{\eta}\right)\right.$, rand $\left.\left(\Psi_{\eta}^{\prime}\right)\right)$, where $\operatorname{rand}(\Psi)$ denotes a random permutation in space and time of the states of a pattern $\Psi$.

This definition proved to be inadequate in general since in situations in which the input stimulus directly altered the states of individual agents, it did not distinguish between clustering which was a direct result of the stimulus and clustering which was due to an effect of the dynamics of interaction among the agents. The use of the term TIGoRS has been refined to refer to only those situations in which there is an enhanced clustering above and beyond the input effect and due to the system dynamics. Figure 1 provides an example in which clustering occurs as the sampling rate increases in the absence of TIGoRS. The near linear relationship between stimulus rate and Hamming distance indicates that the correspondence is driven entirely by the stimulus. Figure 2 on the other hand provides an example of TIGoRS. The Hamming distance response is bimodal and non-linear, consisting of an initial region of near linear and steep decline, followed by a transition region, followed a region with a much slower, also near linear decline. The initial response reflects the effect of the internal dynamic of the system, the later response that of the stimulus alone.

Three classes of cellular automata have been studied. Driven cellular automata [17] are standard finite deterministic cellular automata in which a transient temporal pattern of states is randomly sampled and input into the cellular automaton, which is then allowed to evolve under its natural dynamic. Many classes of driven cellular automata exhibited pseudo TIGoRS (pure input driven correspondence) but cases of true TIGoRS were observed. Driven cellular automata have a rigid dynamic. Cocktail party automata [18] are adaptive, allowing for changes in the rule space of the automaton. Briefly, at time $t$, a cell $A$ with neighbourhood $N$ updates its state to $s$ and then checks throughout to lattice to observe which states were chosen by those cells whose neighbourhoods had the same values as $N$. If the majority of cells chose state $s$, then the cell updates 


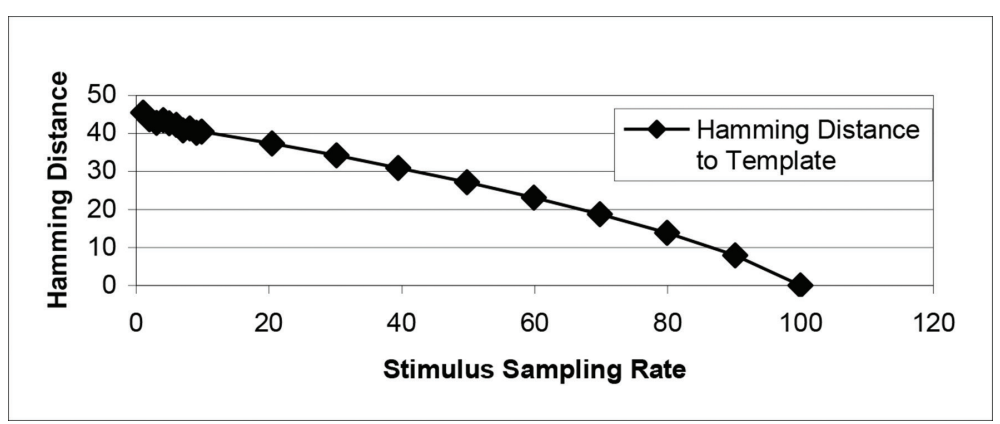

Figure 1: Absence of TIGoRS (Driven C.A. 41, Input 70).

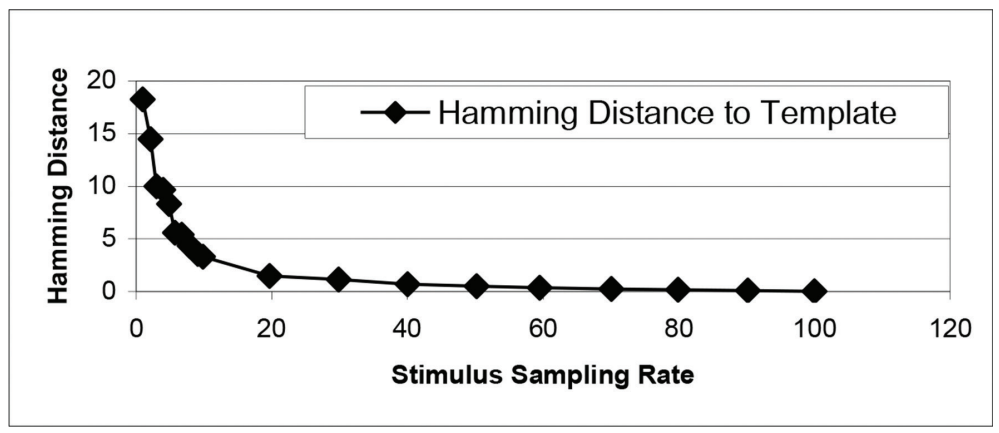

Figure 2: Presence of TIGoRS (Dispositional C.A. 41, Input 70).

its rule, assigning the state $s$ ' to the neighbourhood value $N$. Dispositional cellular automata [19] lie somewhat between driven and cocktail parry automata. The rule space is again dynamic but local. If a given cell at time $t$ receives an input, $s$, then it updates its rule so that a state $s$ will be generated whenever the neighbourhood matches the current neighbourhood of the cell. For dispositional and cocktail party automata, both the state space and the rule space change as the automaton evolves.

\section{COMPUTATIONAL COMPETENCE AND TRANSIENT LANGUAGE}

An understanding of TIGoRS requires some familiarity with the formal notions of computational competence and computational performance [13]. Computational competence refers to the ability or capability of the system under scrutiny to carry out the computation under consideration. Computational performance refers to the actual mechanism by which the computation is carried out.

Computational competence requires

1. a specification, in advance, of the environmental context in which the computation is to take place,

2. a specification of the features of the environment which will be manipulated by the observer,

3. a specification of the behaviours to be exhibited which indicate that the required computation did indeed take place, and

4. a specification of the duration within which this task is to be performed. 
The determination of computational competence consists first of specifying some set of initial conditions together with the environmental constraints to be imposed by the experimenter. Next, the system-environment pair is allowed to evolve over the allotted time. Finally, the experimenter observes whether or not the subsequent behaviours of both the environment and the organism lie within the previously assigned sets. If so, then the requisite computation took place, otherwise not.

Computational performance requires

1. that the system satisfy the conditions of computational competence for the computation in question and

2. that the system be physically capable of implementing this computational computation in reality using whatever means are appropriate to the organism.

There is no assumption as to the form of the process underlying this performance.

The transient language formulation of a dynamical system was introduced to address questions related to computational competence and performance as well as TIGoRS [15-17]. Classically, a dynamical system is described by a set of instantaneous states which forms the state space $S$ together with a dynamics, defined by a mapping $f_{t}: T \times S \rightarrow S$ where $t \in T$ is a time parameter (which may be discrete or continuous) such that for any initial state $x, f_{t}(x)=x_{t}$ and $f_{t} f_{t^{\prime}}=f_{t+t^{\prime}}$. A trajectory in $\mathrm{S}$ from an initial state $x$ is a path of the form $\left\{f_{t}(x) \mid t \in T\right\}$

In the transient language formulation, the set $P$ of all short duration transients forms the state space and the trajectory of a dynamical system is represented as a sequence of finite duration transients. Each transient is thus a trajectory of the form $\Pi_{k}(x)=\left\{f_{t}(x) \mid t \in(0, k)\right\}$. The dynamic is constructed on $P$ by defining a map $g: T \times P \rightarrow P$ such that for any transient $\Pi, g_{t}(\Pi)=\Pi_{t}$ where $\pi_{t} \in P$ and has duration $t$. These short duration transients can be thought of as letters in an alphabet so that a trajectory becomes a sequence of letters, much like a word. The complex system acts as a transducer, converting stimulus 'words' into response 'words' according to some emergent 'grammar'.

In a typical experiment there will be an open set of stimuli $Q$ representing the actions of the experimenter and the effects of the environment, an open set $R$ of responses deemed representative of the appropriate response or correct computation, and some set $\Sigma$ of possible initial histories. The system $\Delta$ is competent to carry out the required behaviour or computation if the set of responses to the stimulus $Q, \Delta(\Sigma, Q)$, satisfies $\Delta(\Sigma, Q) \subset R$.

TIGoRS provides a mechanism which can create such a pairing for at least a subset of all possible stimuli available to a complex system. Stimuli that are capable of inducing TIGoRS at acceptable levels of input (which must be specified according to the particular circumstances of the situation) are termed salient. A discussion of salience and its implications appears elsewhere [19]. The stimulus-response connection using TIGoRS is an example of a 'good enough' information processing paradigm typical of naturally occurring computational systems [13].

Automata possessing architectures and dynamics consistent with these ideas have been called Sulis machines (Dufort \& Lumsden [21]).

\section{RESULTS}

The combinatorics of cellular automata provides a daunting challenge to formal and experimental analysis. A simple 2-state 3 neighbour automaton having 100 cells evolved for just 450 time steps has a pattern space of some $2^{45000}$ patterns. Each cell takes one of 256 possible rules so at any time 
the rule configuration of the automaton is but one of $256^{100}$ possibilities. In spite of this, classifications of cellular automata based on the symmetry properties of the patterns generated under autonomous evolution have been made. One such classification is: uniform, fixed, vertical, diagonal, complex and chaotic [16]. It is impossible to exhaustively search the entire states space. Instead, a smaller sample must be chosen, usual derived from the symmetry structure of the automata and of the stimulus patterns.

As an illustration of the essential qualitative features of TIGoRS, a series of simulations of the three types of cellular automata (driven, dispositional and cocktail party) were carried out for 2 state 3 neighbour systems having 100 cells and observed for 450 time steps. In previous studies TIGoRS generally occurred within this duration. Each automaton was initialized with a uniform rule configuration and a random initial state with $10 \%$ of the cells having state 1 . The initial rules were 7 (diagonal), 14 (diagonal), 37 (vertical), 40 (uniform), 41 (complex), 43 (diagonal), 44 (fixed), 45 (chaotic). The input patterns were taken as outputs from autonomous cellular automata having rules 70 (vertical), 72 (fixed), 73 (complex), 74 (diagonal), 75 (chaotic).

The sampling template used to generate the input was randomly generated and distinct for each automaton simulated. A fixed input pattern was sampled according to the sampling template and whenever the pattern was sampled, the corresponding cell had its state changed to that of the input pattern at that site. After sampling, the automaton was allowed to update under its own dynamic. Simulations were carried out for durations from 50-450 times steps in 50 step increments but only results for the 450 time step are reported here.

In order to avoid problems of pseudo-TIGoRS, a new measure of TIGoRS was developed in order to detect only genuine TIGoRS. Using a fixed sampling template corresponding to a fraction $p$ of pattern sites, a fraction $p$ of automaton cells will automatically match the pattern and the responses of other identically stimulated automata. We are interested, however, in the excess synchronization provided by the dynamics of the system itself, and so need to eliminate the input contribution. Thus, the excess synchronization (efficacy) of TIGoRS was defined as follows: $E(p)=-P+H(0)-H(p)$, where $P$ is the percentage of pattern cells sampled, $H(0)$ is the percentage Hamming distance between the current automaton response and the template response in the absence of input and $H(p)$ is the percentage Hamming distance between the template response and the current automaton response under input by pattern $p$ with sampling $P$.

Three different cellular automata were studied: driven cellular automata, dispositional cellular automata and cocktail party automata. Each class of automata was simulated for the 40 possible input-rule pairings. Of the 40 combinations studied, genuine TIGoRS appeared in 3 cases for driven cellular automata, 28 cases for dispositional cellular automata and 21 cases for cocktail party automata. It should be noted that when the same sampling template is used for all of the simulations, the cocktail party automaton showed TIGoRS in 36 cases. The cocktail party automaton appears to be more discriminating, and there are fewer patterns capable of forcing a global binding of automaton output. It is clear that dispositional and adaptive systems are better able to support emergent processes such as TIGoRS than fixed systems although at the expense of a loss of simple causal relationships between low- and high-level processes.

In the case of driven automata, in two cases (14-70,37-72) TIGoRS applied only to the matching of automaton responses, not of automaton response and pattern. This also occurred in two cases (14-74, 40-73) for dispositional cellular automata, and not at all for the cocktail party automata. The converse situation occurred three times for the dispositional automata $(14-70,14-72,44-72)$ and eleven times for the cocktail party automaton $(7-72,14-72,37-72,37-73,40-72,41-73,41-74$, 43-72, 43-73, 44-72, 45-72). The cocktail party automaton behaved more as a pattern transducer and less like a pattern replicator. 


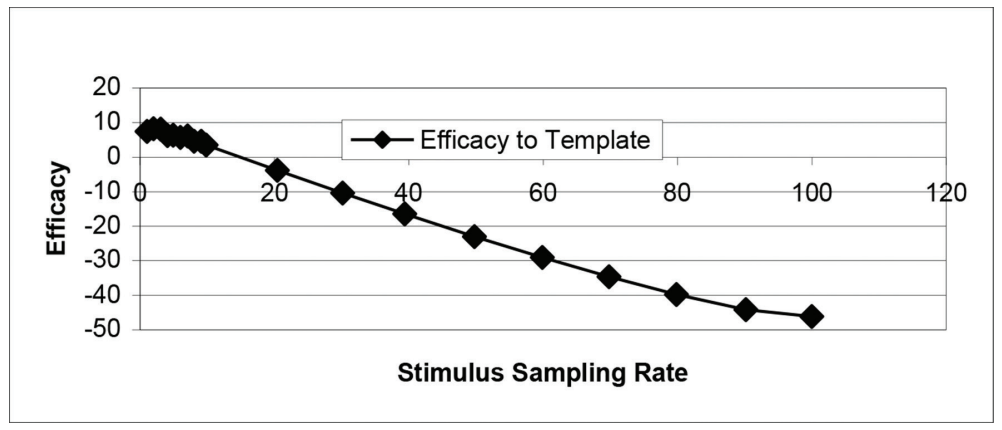

Figure 3: Absence of TIGoRS (Driven C.A. 41, Input 70).

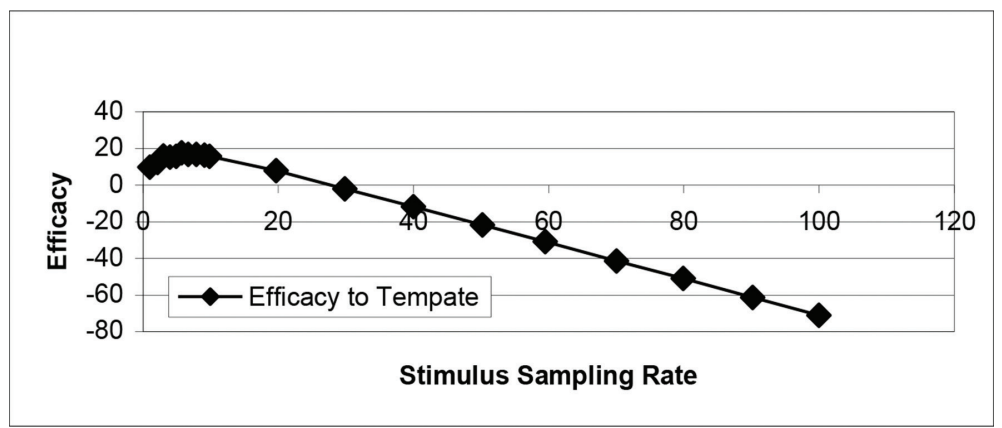

Figure 4: Presence of TIGoRS (Dispositional C.A. 41, Input 70).

Figures 3 and 4 depict the change in efficacy between different automata runs as a function of sampling frequency in the presence of TIGoRS.

The efficacy curve is approximately linear in the case of pseudo-TIGoRS, steadily declining as the sampling rate increases, while it is definitely non-linear in the case of genuine TIGoRS. The efficacy rises rapidly for the first few sampling frequencies, achieving a positive maximum value and then steadily declines as the stimulating frequency increases further. This suggests the presence of two dynamical regimes: an initial regime in which the automaton dynamics augments the effect of the pattern stimulus resulting in rapid synchronizing effect, followed by a second regime in which the bulk of the synchronization is passive arising as a direct result of pattern sampling. These figures provide convincing evidence that TIGoRS is a real dynamical response of the system and is not merely an artefact of the stimulus paradigm.

In both cases the same complex rule (71) was used for the initial rule configuration, while a diagonal pattern (70) was used to provide the input. This pattern has a complicated multi-frequency structure which propagates around the automaton cells. The difference lies in the dynamics - standard cellular automaton in the first case and dispositional in the second. This shows one benefit of the dispositional dynamic. A cocktail party automaton with the same initial rule configuration also exhibits TIGoRS under this input pattern, and the maximal excess synchronization occurs two time steps earlier than for the dispositional automaton.

A direct comparison of the three automata types can be seen in Figs 5 and 6 which depict the changes in Hamming distance and excess synchronization to the pattern for the same initial rule (41) and input pattern (70). 


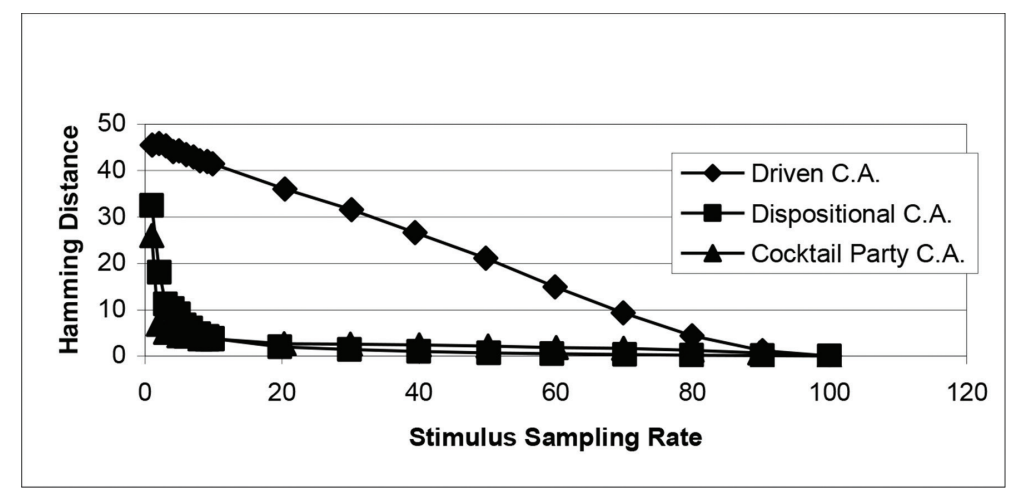

Figure 5: Hamming Distance to Pattern. Comparisons of Three Automata (41-70).

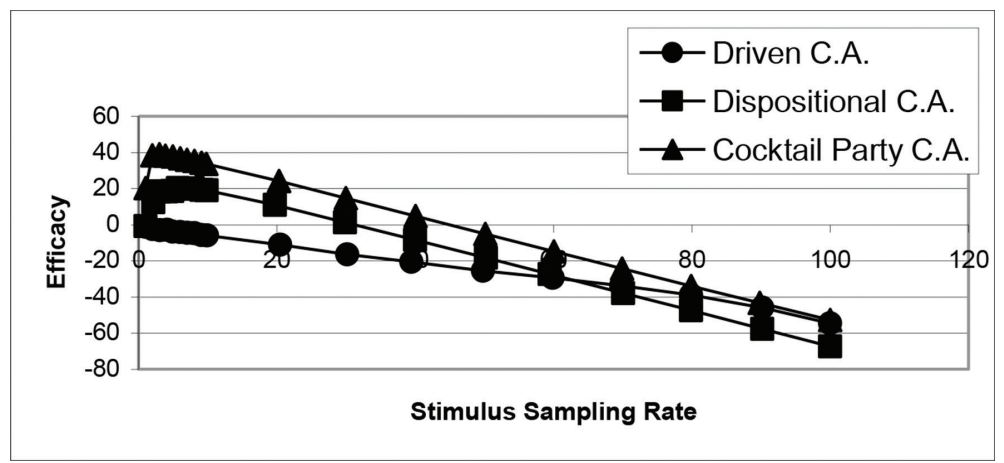

Figure 6: Efficacy to Pattern. Comparisons of Three Automata (41-70).

\section{CONCLUSIONS}

The phenomenon of TIGoRS is ubiquitous across a wide range of complex systems models and this may be true of naturally occurring computational systems as well [20]. TIGoRS appears to be a emergent non-linear reaction to the presence of an external stimulus which depends upon the particular underlying dynamic of the system. TIGoRS enables a complex system to produce an emergent response to an environmental stimulus that is stable across a broad range of initial, rule and stimulus conditions. TIGoRS provides a means through which information is stored, not as an invariant property of the system, but as a dynamical response. The system extracts salient information out of the environment to construct its response rather than storing the necessary information within its own structure. This is particularly true of systems evolving according to a dispositional dynamic in which the presence of an external input stimulus alters not just the state of the system but its internal underlying dynamic as well.

TIGoRS is a form of synchronization that may have wide spread expression throughout the biological and social realms and is deserving of further scrutiny.

\section{REFERENCES}

[1] Wilson, E.O. \& Holldobler, B., The Ants, Belknap Press: Cambridge, 1990.

[2] Guastello, S.J., Pincus, D. \& Gunderson, P.R., Electrodermal arousal between participants in a conversation: nonlinear dynamics for linkage effects. Nonlinear Dynamics Psychology and Life Sciences, 10, pp. 365-399, 2006. 
[3] Aertsen, A.D. \& Arndt, M., Response synchronization in the visual cortex. Current Opinion in Neurobiology, 3(4), pp. 586-594, 1993.

http://dx.doi.org/10.1016/0959-4388(93)90060-C

[4] Eckhorn, R., Bauer, R., Jordan, W., Kruse, W., Munk, M. \& Reitboeck, H., Coherent oscillations: a mechanism of feature linking in the visual cortex. Multiple electrode and correlation analyses in the cat. Biological Cybernetics, 60(2), pp. 121-130, 1988.

http://dx.doi.org/10.1007/BF00202899

[5] Mason, A., Nicoll, A. \& Stratford, K., Synaptic transmission between individual pyramidal neurons of the rat visual cortex in vivo. The Journal of Neuroscience, 11(1), pp. 72-84, 1991.

[6] Mainen, Z.F. \& Sejnowski, T.J., Reliability of spike timing in neocortical neurons. Science, 268(5216), pp. 1503-1506, 1995.

http://dx.doi.org/10.1126/science.7770778

[7] Shadlen, M.N. \& Newsome, W.T., Noise, neural codes and cortical organization. Current Opinion in Neurobiology, 4, pp. 569-579, 1994.

http://dx.doi.org/10.1016/0959-4388(94)90059-0

[8] Barnes, C.A., Suster, M.S., Shen, J. \& McNaughton, B.L., Multistability of cognitive maps in the hippocampus of old rats. Nature, 388, pp. 272-275, 1997.

http://dx.doi.org/10.1038/40859

[9] Jung, M.W. \& McNaughton, B.L., Spatial selectivity of unit activity in the hippocampal granular layer. Hippocampus, 3(2), pp. 165-182, 1993.

http://dx.doi.org/10.1002/hipo.450030209

[10] Quirk, G.J., Mueller, R.U. \& Kubie, J.L., The firing of hippocampal place cells in the dark depends on the rat's recent experience. The Journal of Neuroscience, 10(6), pp. 2008-2017, 1990.

[11] Sulis, W., Collective intelligence: observations and models. In Chaos and Complexity in Psychology, eds. S. Guastello, M. Koopmans \& D. Pincus, Cambridge University Press: Cambridge, 2009.

http://dx.doi.org/10.1109/ijenn.1992.227138

[12] Trofimova, I.N., Sociability, diversity, and compatibility in developing systems: EVS approach. In Formal Descriptions of Developing System, eds. J. Nation, I. Trofimova, J. Rand \& W. Sulis, Boston: Kluwer, 2003.

[13] Sulis, W., Naturally occurring computational systems. World Futures, 39(4), pp. 229-241, 1993.

[14] Sulis, W., Tempered neural networks. In Proceedings of the International Joint Conference on Neural Networks '92, Vol. III, IEEE Press: Baltimore, 1992.

[15] Sulis, W., Emergent computation in tempered neural networks: dynamical automata. In Proceedings of the World Congress on Neural Networks '93 Vol. IV 449, Lawrence Erlbaum: New York, 1993.

[16] Sulis, W., Emergent computation in tempered neural networks: computation theory. In Proceedings of the World Congress on Neural Networks '93, Vol. IV 452, Lawrence Erlbaum: New York, 1993.

[17] Sulis, W., Driven cellular automata. In 1993 Lectures in Complex Systems, eds. D. Stein \& L. Nadel, Addison-Wesley: New York, 1993.

[18] Sulis, W., Driven cellular automata, adaptation, and the binding problem. In Advances in Artificial Life, Lectures Notes in Artificial Intelligence 929, eds. F. Moran, A. Moreno, J. Merelo \& P. Chacon, Springer-Verlag: New York, 1995. http://dx.doi.org/10.1007/3-540-59496-5_346 
[19] Sulis, W., Synchronization, TIGoRS, and information flow in complex systems. Nonlinear Dynamics Psychology and Life Science April, In Press, 2016.

[20] Sulis, W., Archetypal dynamics, emergent situations and the reality game. Nonlinear Dynamics Psychology and Life Science, 14(3), pp. 209-238, 2010.

[21] Dufort, P.A. \& Lumsden, C.J., Dynamics, complexity and computation. In Physical Theory in Biology: Foundations and Exploration, eds. C.J. Lumsden, W.A. Brandts \& L.E.H. Trainor, World Scientific Press: Singapore, 1997. http://dx.doi.org/10.1142/9789812819598_0004 